\title{
Corrigendum
}

\section{Corrigendum to "Risk Factors for Fracture in Diabetes: The Canadian Multicentre Osteoporosis Study"}

\author{
Lisa-Ann Fraser, ${ }^{1,2}$ Alexandra Papaioannou, ${ }^{3,4}$ Jonathan D. Adachi, ${ }^{4}$ \\ Jinhui $\mathrm{Ma}^{3}$ and Lehana Thabane ${ }^{3,5}$ \\ ${ }^{1}$ Department of Medicine, University of Western Ontario, 339 Windermere Road, London, ON, Canada N6A 5A5 \\ ${ }^{2}$ Division of Endocrinology and Metabolism, St. Joseph's Hospital, 268 Grosvenor Street, London, ON, Canada N6A 4V2 \\ ${ }^{3}$ Department of Epidemiology and Biostatistics, McMaster University, 1280 Main Street West, Hamilton, ON, Canada L8S 4 K1 \\ ${ }^{4}$ Department of Medicine, McMaster University, 1280 Main Street West, Hamilton, ON, Canada L8S 4K1 \\ ${ }^{5}$ Centre for Evaluation of Medicines, St. Joseph's Healthcare, 50 Charlton Avenue East, Hamilton, ON, Canada L8N 4A6
}

Correspondence should be addressed to Alexandra Papaioannou; papaioannou@hhsc.ca

Received 6 June 2017; Accepted 8 June 2017; Published 12 July 2017

Copyright (C) 2017 Lisa-Ann Fraser et al. This is an open access article distributed under the Creative Commons Attribution License, which permits unrestricted use, distribution, and reproduction in any medium, provided the original work is properly cited.

In the article titled "Risk Factors for Fracture in Diabetes: The Canadian Multicentre Osteoporosis Study" [1], there was an error regarding the FRAX ${ }^{\circledR}$ tool, which should be clarified as follows.

The article notes: "The WHO fracture risk assessment tool (FRAX), a commonly used fracture prediction tool, has been found to underestimate fracture risk in the diabetic population [10]." However, the World Health Organization (WHO) did not develop, test, or endorse the FRAX tool or its recommendations [2]. The metabolic bone disease unit at the University of Sheffield that developed FRAX was a WHO Collaborating Centre from 1991 to 2010, but treatment guidelines must undergo a formal process before they can be endorsed by the WHO.

\section{References}

[1] L. Fraser, A. Papaioannou, J. D. Adachi, J. Ma, and L. Thabane, "Risk factors for fracture in diabetes: the canadian multicentre osteoporosis study," ISRN Epidemiology, vol. 2013, Article ID 249874, 6 pages, 2013.

[2] N. Ford, S. L. Norris, and S. R. Hill, "Clarifying WHO's position on the FRAX ${ }^{\circledR}$ tool for fracture prediction," Bulletin of the World Health Organization, vol. 94, no. 12, article 862, 2016. 


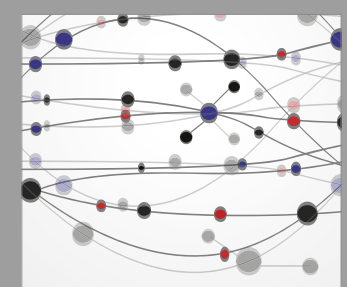

The Scientific World Journal
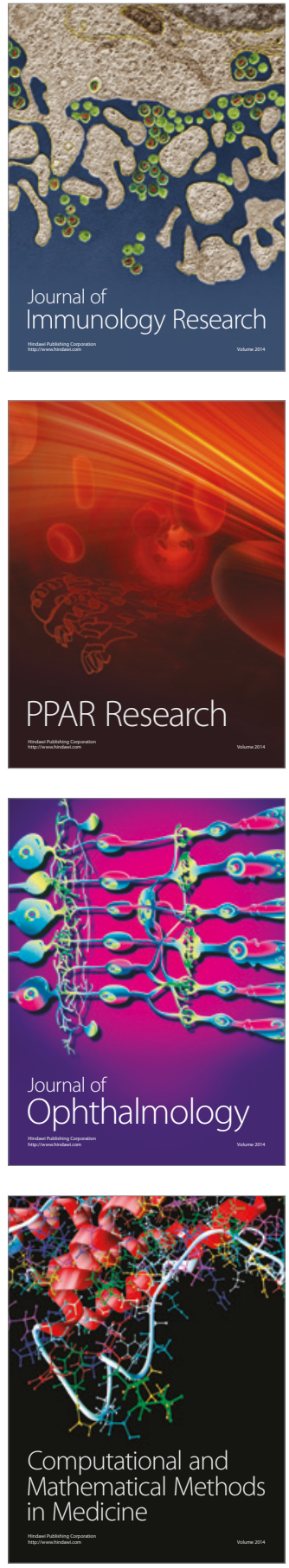

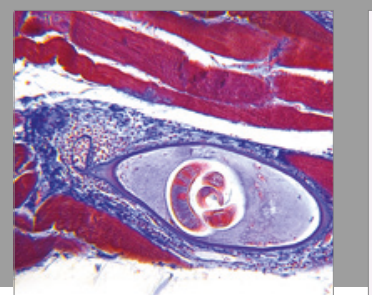

Gastroenterology Research and Practice
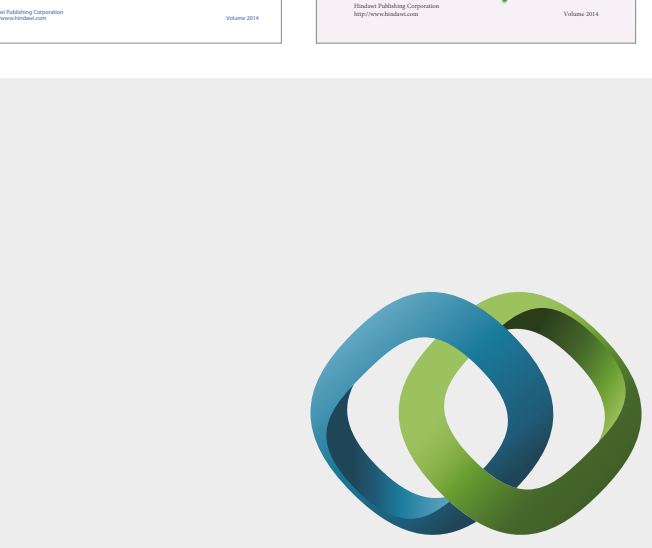

\section{Hindawi}

Submit your manuscripts at

https://www.hindawi.com
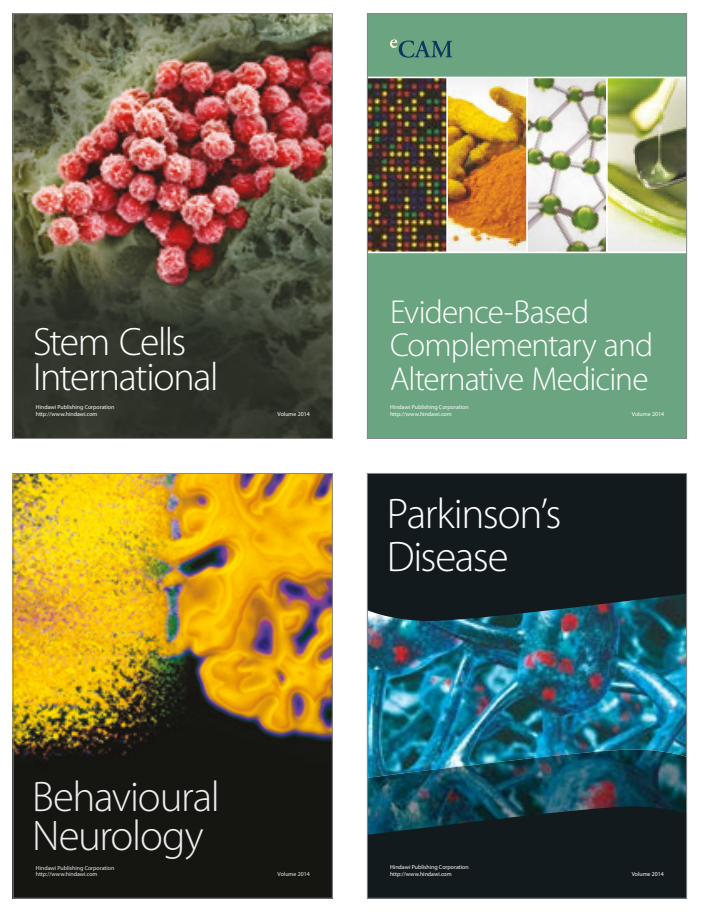
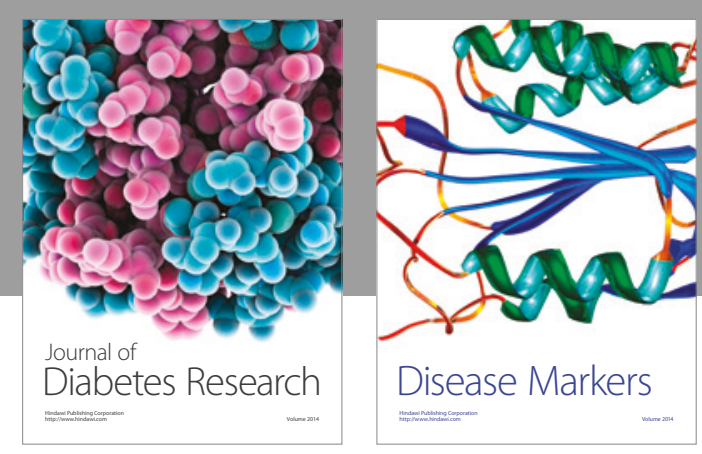

Disease Markers
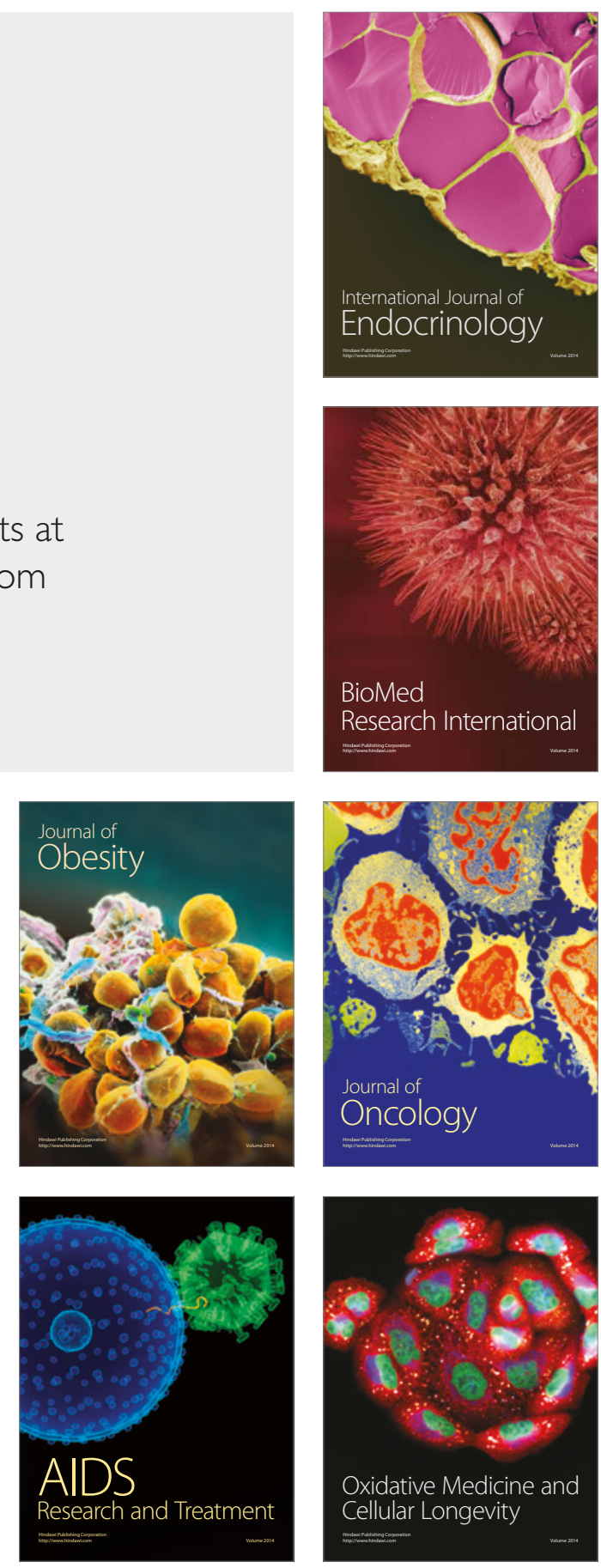